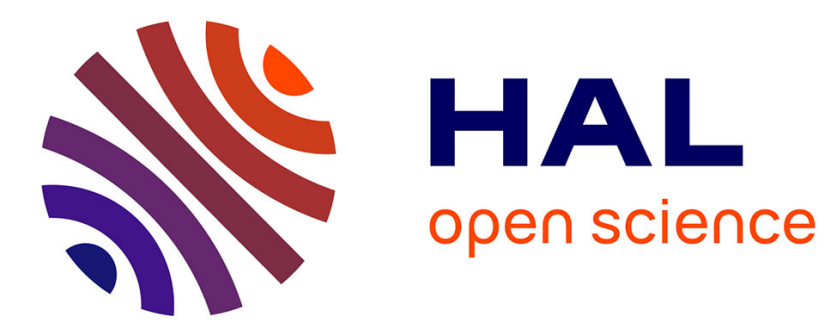

\title{
The Structure and Torsional Dynamics of Two Methyl Groups in 2-Acetyl-5-methylfuran as Observed by Microwave Spectroscopy
}

\author{
Vinh Van, Wolfgang Stahl, Ha Vinh Lam Nguyen
}

\section{To cite this version:}

Vinh Van, Wolfgang Stahl, Ha Vinh Lam Nguyen. The Structure and Torsional Dynamics of Two Methyl Groups in 2-Acetyl-5-methylfuran as Observed by Microwave Spectroscopy. ChemPhysChem, 2016, 17 (20), pp.3223-3228. 10.1002/cphc.201600757 . hal-03183106

\section{HAL Id: hal-03183106 https://hal.science/hal-03183106}

Submitted on 26 Mar 2021

HAL is a multi-disciplinary open access archive for the deposit and dissemination of scientific research documents, whether they are published or not. The documents may come from teaching and research institutions in France or abroad, or from public or private research centers.
L'archive ouverte pluridisciplinaire HAL, est destinée au dépôt et à la diffusion de documents scientifiques de niveau recherche, publiés ou non, émanant des établissements d'enseignement et de recherche français ou étrangers, des laboratoires publics ou privés. 


\section{The structure and torsional dynamics of two methyl groups in 2- acetyl-5-methylfuran as observed by microwave spectroscopy}

\begin{abstract}
Vinh Van ${ }^{[b]}$, Wolfgang Stah ${ }^{[b]}$, and Ha Vinh Lam Nguyen ${ }^{*}[a]$
\end{abstract}
expected that splittings arising from the internal rotations of both methyl groups can be resolved. The barrier heights of such large amplitude motions (LAM) are mainly determined by steric and electronic effects. While the steric influence is rather local, the electronic contribution can arise from quite distant sources in the molecule, especially when conjugated double bonds and/or aromatic systems are involved. Predicting torsional barriers is challenging because chemical intuition often fails and quantum chemical calculations are still rather inaccurate $[5,6]$. On the other hand, microwave spectroscopy yields highly accurate torsional barriers. Moreover, two different AMF conformers exist, where at least the acetyl methyl group is exposed to different steric environments, while the ring methyl group is almost unaffected.

\section{Quantum Chemical Calculations}

From the structural point of view, AMF is rather rigid because the conjugated double bonds in the five-membered ring force the furan frame to be planar. Therefore, the conformational landscape is solely determined by a rotation about the $\mathrm{C}_{4}-\mathrm{C}_{12}$ bond (for atom numbering see Figure 1). By varying the dihedral angle $\varphi_{1}=\angle\left(\mathrm{O}_{11}, \mathrm{C}_{4}, \mathrm{C}_{12}, \mathrm{O}_{13}\right)$ in a grid of $2^{\circ}$ while all other geometry parameters were optimized at the MP2/6-311++G(d,p) level of theory using the GAUSSIAN 09 package [7], we obtained a potential energy curve with two minima. The calculated energies were parameterized using a Fourier expansion with the corresponding coefficients given in Table S-1 in the Supporting Information. Using these Fourier coefficients, the potential energy curve was drawn as depicted in Figure 2. Geometries at the minima were fully re-optimized using the HF, B3LYP, M06-2X, MP2, and CCSD methods in combination with various Pople and Dunning basis sets $[8,9]$ to compare the rotational constants and check for convergence. The predicted rotational constants are collected in Table S-2. If not otherwise stated, all values given from now on will refer to those calculated at the MP2/6-311++G(d,p) level of theory. Figure 1 illustrate the fully optimized conformers; their Cartesian coordinates are given in Table S-3. The conformer at $\varphi_{1}=180^{\circ}$, called the trans conformer henceforth, is with zero-point energy correction 4.19 $\mathrm{kJ} \cdot \mathrm{mol}^{-1}$ lower in energy than the so-called cis conformer $\left(\varphi_{1}=0^{\circ}\right)$.
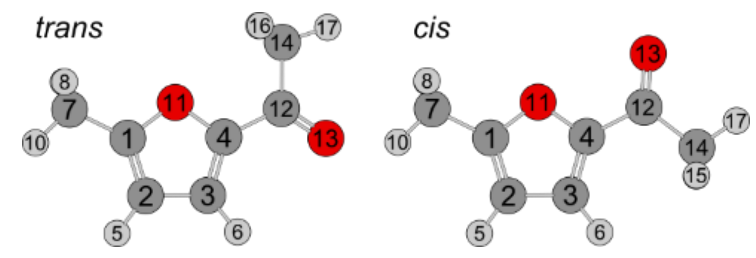

Figure 1. Two conformers of AMF optimized at the MP2/6-311++G(d,p) level of theory.

\footnotetext{
Dr. H. V. L. Nguyen

Laboratoire Interuniversitaire des Systèmes Atmosphériques (LISA) CNRS UMR 7583, Université Paris-Est Créteil, Université Paris Diderot

61 avenue du Général de Gaulle, F-94010 Créteil cedex, France E-mail: lam.nguyen@lisa.u-pec.fr

[b] Vinh Van M. Sc., Prof. Dr. Wolfgang Stahl Institute of Physical Chemistry

RWTH Aachen University

Landoltweg 2, D-52074 Aachen, Germany
}

Supporting information for this article is given via a link at the end of the document. 


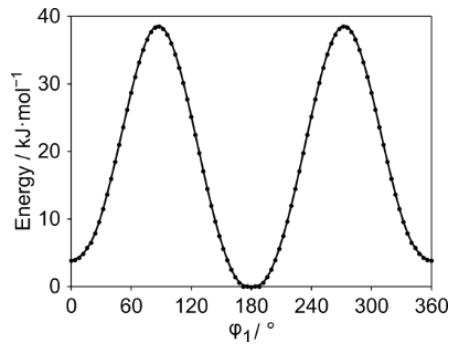

Figure 2. The potential energy curve obtained by varying the dihedral angle $\varphi_{1}$ $=\angle\left(\mathrm{O}_{11}, \mathrm{C}_{4}, \mathrm{C}_{12}, \mathrm{O}_{13}\right)$ in a grid of $2^{\circ}$ and optimized at the MP2/6-311++G(d,p) level of theory. Relative energies are given with respect to the lowest energy configuration with its absolute energy $\mathrm{E}=-420.930421 \mathrm{Hartree}$. The trans conformer is located at $\varphi_{1}=180^{\circ}$, the cis conformer at $0^{\circ}$
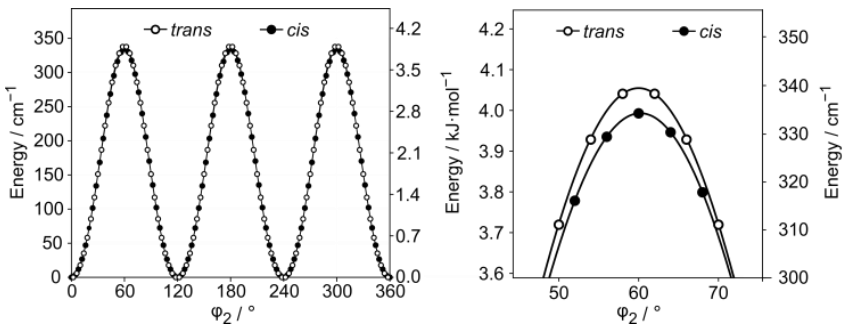

Figure 3. The potential energy curves of the trans and the cis conformer of AMF obtained by rotating the ring methyl group about the $\mathrm{C}_{1}-\mathrm{C}_{7}$ bond. The dihedral angle $\varphi_{2}=\angle\left(\mathrm{C}_{2}, \mathrm{C}_{1}, \mathrm{C}_{7}, \mathrm{H}_{8}\right)$ was varied in a grid of $2^{\circ}$, while all other molecular parameters were optimized at the MP2/6-311++G(d,p) level. Relative energies with respect to the lowest energy conformations with the absolute energies $E=-420.930421$ and -420.928812 Hartree for the trans and the cis conformer, respectively, are used. The barrier heights are $340 \mathrm{~cm}^{-1}$ for the trans and $334 \mathrm{~cm}^{-1}$ for the cis conformer.

In spite of its relatively simple structure, the internal dynamic of AMF is challenging because of the LAMs of two inequivalent methyl groups, which cause all rotational transitions to split into quintets [10]. The barrier heights of the ring methy and the acetyl methyl group were calculated by varying the dihedral angles $\varphi_{2}=\angle\left(\mathrm{C}_{2}, \mathrm{C}_{1}, \mathrm{C}_{7}, \mathrm{H}_{8}\right)$ and $\varphi_{3}=\angle\left(\mathrm{O}_{13}, \mathrm{C}_{12}, \mathrm{C}_{14}, \mathrm{H}_{15}\right)$ respectively, in a grid of $2^{\circ}$. A rotation of $120^{\circ}$ was sufficient due to the three-fold symmetry of the methyl groups. The potential energy curves of the ring methyl group are shown in Figure 3; the corresponding coefficients in Table S-1 in the Supporting Information. For the trans conformer, we found $V_{3}$ potentials of $340 \mathrm{~cm}^{-1}$ for the ring methyl group and $241 \mathrm{~cm}^{-1}$ for the acetyl methyl group with no significant $V_{6}$ contributions. The respective values for the cis conformer are $334 \mathrm{~cm}^{-1}$ and $207 \mathrm{~cm}^{-1}$.

The potential energy curves for a rotation of the acetyl methyl groups are depicted in Figure 4 . Here, we expected $V_{6}$ contributions for the trans conformer due to a more symmetrical environment of the acetyl methyl rotor in relation to both oxygen atoms. Unfortunately, an unusual discontinuity occurred in the minimum regions of the potential curves of this conformer during the rotation of the acetyl methyl group. Therefore, the data set cannot be fitted well. By comparing the two geometries at the discontinued place, we found that (i) only the methyl group was twisted, while all other dihedral angles remain the same, and (ii) one hydrogen atom approaches the eclipse position of the oxygen atom at this place (see Figure S-1 in the Supporting Information). The barrier height of $241 \mathrm{~cm}^{-1}$ obtained from $E\left(60^{\circ}\right)$ minus $E\left(120^{\circ}\right)$ is essentially the same as the energy difference between the optimized minimum and the transition state.

As an alternative, geometry optimizations to a first order transition state of the methyl groups were performed at various levels of theory using the Berny algorithm to calculate the barriers to internal rotations [11]. The $V_{3}$ potentials predicted at different levels of theory and the angles between the internal rotor axes and the principal axes are summarized in Table S-2.

Finally, a two-dimensional potential energy surface (2DPES) depending on $\varphi_{2}$ and $\varphi_{3}$ were performed by varying these two dihedral angles in a grid of $10^{\circ}$ to study the coupling between the two LAMs of the methyl groups. Due to symmetry, only data points in the range from $\varphi_{2}$ and $\varphi_{3}=0^{\circ}$ to $120^{\circ}$ are needed. The potential energies were parameterized with a $2 \mathrm{D}$ Fourier expansion based on terms representing the correct symmetry of the angles $\varphi_{2}$ and $\varphi_{3}$. The corresponding coefficients are given in Table S-1 in the Supporting Information. The PES illustrated in Figure 5 exhibits almost no potential coupling terms between $\varphi_{2}$ and $\varphi_{3}$ for both conformers.

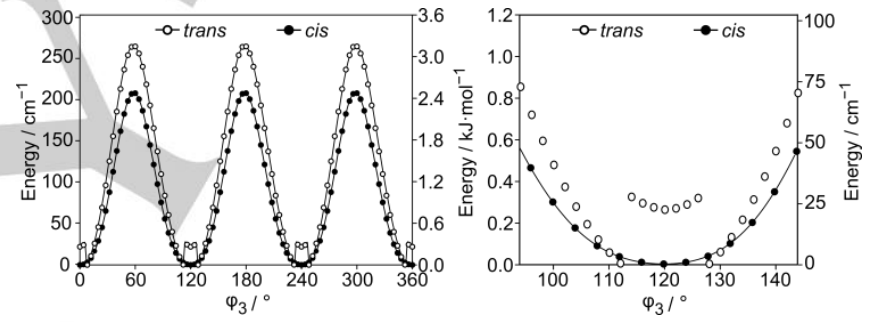

Figure 4. The potential energy curves of the trans and the cis conformer of AMF obtained by rotating the acetyl methyl group about the $\mathrm{C}_{12}-\mathrm{C}_{14}$ bond. The dihedral angle $\varphi_{3}=\angle\left(\mathrm{O}_{13}, \mathrm{C}_{12}, \mathrm{C}_{14}, \mathrm{H}_{15}\right)$ was varied in a grid of $2^{\circ}$, while all other molecular parameters were optimized at the MP2/6-311++G(d,p) level. Relative energies with respect to the lowest energy conformations with the absolute energies $E=-420.930421$ and -420.928812 Hartree for the trans and the cis conformer, respectively, are given. The barrier height is $207 \mathrm{~cm}^{-1}$ for the cis conformer. For the trans conformer, an unusual discontinuity occurred in the minimum regions, as can be recognized in the enlarged scale (right hand side figure)

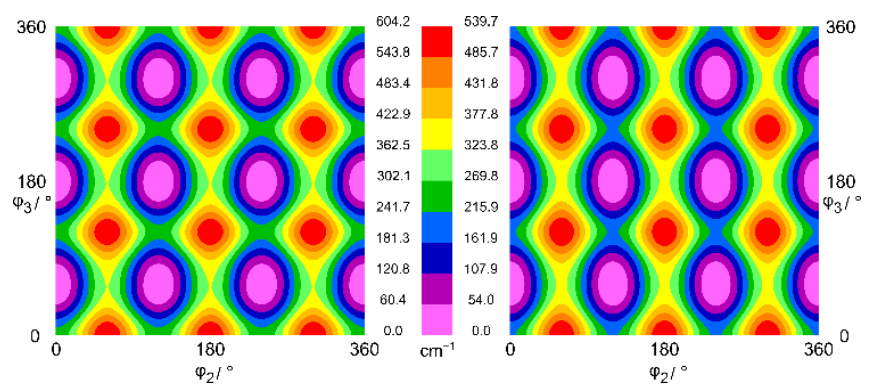

Figure 5. The potential energy surface depending on the dihedral angles $\varphi_{2}=\angle\left(\mathrm{C}_{2}, \mathrm{C}_{1}, \mathrm{C}_{7}, \mathrm{H}_{8}\right)$ and $\varphi_{3}=\angle\left(\mathrm{O}_{13}, \mathrm{C}_{12}, \mathrm{C}_{14}, \mathrm{H}_{15}\right)$ of the trans (left hand side) and the cis conformer (right hand side) of AMF calculated at the MP2/6$311++G(d, p)$ level of theory. $\varphi_{2}$ and $\varphi_{3}$ were varied in a grid of $10^{\circ}$, while all other parameters were optimized. The relative energy maxima are $604.2 \mathrm{~cm}^{-1}$ $\left(7.2 \mathrm{~kJ} \cdot \mathrm{mol}^{-1}\right)$ and $539.7 \mathrm{~cm}^{-1}\left(6.5 \mathrm{~kJ} \cdot \mathrm{mol}^{-1}\right)$ for the trans and the cis conformer, respectively. 


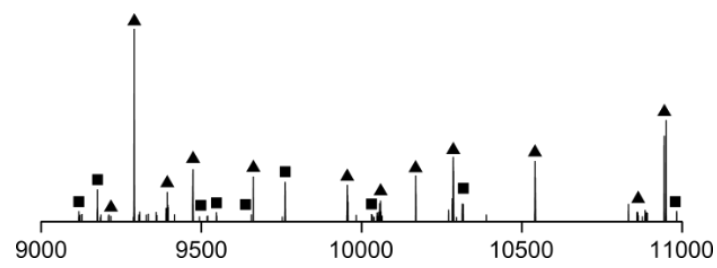

Figure 6. A portion of the broadband scan from 9000 to $11000 \mathrm{MHz}$ of AMF showing that (i) transitions of the trans conformer $(\mathbf{A})$ are much more intense than those of the cis conformer ( $\mathbf{m}$ ), and (ii) only a few unassigned lines are present in the spectrum.

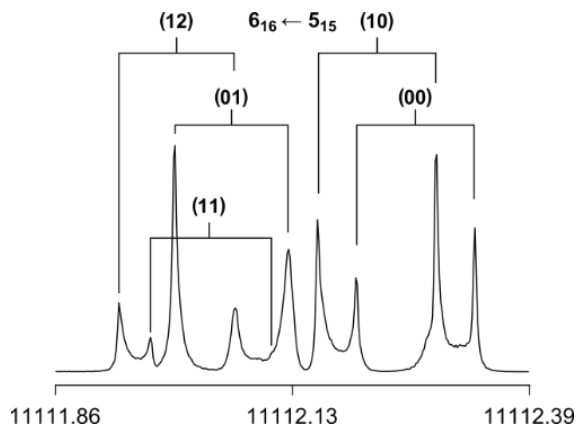

Figure 7. A typical spectrum of the $6_{16} \leftarrow 5_{15}$ transition of the trans conformer of AMF with its (00), (10), (01), (11), and (12) torsional species. The frequency is in $\mathrm{MHz}$; the line width is approximately $13-25 \mathrm{kHz}(\mathrm{FWHH})$, corresponding to a measurement accuracy of $2 \mathrm{kHz}$. The splittings indicated by brackets are due to the Doppler effect. For this spectrum 2000 decays were co-added.

\section{Microwave spectroscopy}

We started the assignment with the spectrum of the more stable trans conformer, which has dipole moment components of 4.14 D, 1.67 D, and 0.00 D in $a-, b$-, and c-direction, respectively. Only $a$ - and $b$-type transitions are expected in the spectrum, and a-type lines should be more intense. The selection rules for $a-$ type lines are $K_{a} K_{c}$ : ee $\leftrightarrow$ eo and oo $\leftrightarrow$ oe and for $b$-type lines $K_{a} K_{c}$ : ee $\leftrightarrow$ oo and oe $\leftrightarrow$ eo (e = even, o = odd) [12]. At the beginning, we neglected the internal rotation effects and considered AMF as a rigid rotor. A prediction carried out with the program XIAM [13] indicated a relatively dense spectrum in the frequency range from 9.0 to $13.0 \mathrm{GHz}$. A broadband scan was recorded in this region, where overlapping spectra in a step size of $0.25 \mathrm{MHz}$ were automatically recorded. Figure 6 shows a portion of the scan. All lines indicated in the scan were remeasured at higher resolution. A typical high resolution spectrum is shown in Figure 7.

The $J=5 \leftarrow 4$ a-type transitions with $K_{\mathrm{a}}=0,1$ and the $b$ type $6_{06} \leftarrow 5_{15}$ and $7_{07} \leftarrow 6_{16}$ as well as $4_{14} \leftarrow 3_{03}, 5_{15} \leftarrow 4_{04}$, and $6_{16} \leftarrow 5_{05}$ transitions were readily assigned. Each transition splits into five torsional component $\left(\sigma_{1} \sigma_{2}\right)=(00),(01),(10),(11)$, and (12) because of the LAMs of two methyl groups [10]. The notations $\sigma_{1}$ and $\sigma_{2}$ refer to the ring methyl rotor and the acetyl methyl rotor, respectively. The assignment of these lines enabled us to predict and find further (00) transitions.

In the next step, we took the methyl internal rotations into consideration and predicted a two-top fit using the rotational
Table 1. Molecular parameters of the trans and the cis conformer of AMF as obtained by the program XIAM. Top 1 refers to the ring methyl group, top 2 to the acetyl methyl group.

\begin{tabular}{|c|c|c|c|c|c|}
\hline Par. ${ }^{[a]}$ & Unit & trans & $M P 2^{[b]}$ & cis & $M P 2^{[b]}$ \\
\hline $\bar{A}$ & $\mathrm{GHz}$ & $3.699650628(73)$ & 3.638 & $3.9138572(37)$ & 3.843 \\
\hline$B$ & $\mathrm{GHz}$ & $1.125952913(72)$ & 1.120 & $1.09556167(24)$ & 1.089 \\
\hline C & $\mathrm{GHz}$ & $0.872880211(49)$ & 0.866 & $0.86550261(12)$ & 0.858 \\
\hline$D_{J}$ & $\mathrm{kHz}$ & $0.03511(28)$ & 0.03449 & $0.0294(20)$ & 0.03042 \\
\hline$D_{J K}$ & $\mathrm{kHz}$ & $-0.0392(29)$ & -0.04428 & $-0.050(17)$ & -0.03050 \\
\hline$D_{K}$ & $\mathrm{kHz}$ & $1.10(17)$ & 0.7370 & $0.783(71)$ & 0.7145 \\
\hline$d_{1}$ & $\mathrm{kHz}$ & $-0.01066(20)$ & -0.01036 & $-0.0066(14)$ & -0.008137 \\
\hline$d_{2}$ & $\mathrm{kHz}$ & $-0.00124(16)$ & -0.0009489 & $0.00^{[c]}$ & -0.0002910 \\
\hline$V_{3,1}$ & $\mathrm{~cm}^{-1}$ & $369.78(25)$ & 340. & $356.47(39)$ & 334. \\
\hline$F_{0,1}$ & $\mathrm{GHz}$ & $159.40(11)$ & 159.3 & $159.16(16)$ & 159.2 \\
\hline$\angle\left(\mathrm{i}_{1}, a\right)$ & $\circ$ & $143.601(34)$ & 143.11 & $146.523(69)$ & 146.36 \\
\hline$\angle\left(\mathrm{i}_{1}, b\right)$ & $\circ$ & $126.399(34)$ & 126.89 & $123.477(69)$ & 123.64 \\
\hline$\angle\left(\mathrm{i}_{1}, C\right)^{[\mathrm{d}]}$ & $\circ$ & 90.0 & 90.00 & 90.0 & 90.00 \\
\hline$V_{3,2}$ & $\mathrm{~cm}^{-1}$ & $307.78(59)$ & 241 & 212.71(30) & 207 \\
\hline$F_{0,2}$ & $\mathrm{GHz}$ & $160.51(39)$ & 158.0 & $159.87(24)$ & 158.1 \\
\hline$\angle\left(\mathrm{i}_{2}, a\right)$ & $\circ$ & $75.154(31)$ & 78.59 & $-41.012(15)$ & -44.48 \\
\hline$\angle\left(\mathrm{i}_{2}, b\right)$ & $\circ$ & $165.154(31)$ & 168.59 & $48.988(15)$ & 45.52 \\
\hline$\angle\left(\mathrm{i}_{2}, C\right)^{[\mathrm{d}]}$ & & 90.0 & 89.99 & 90.0 & 90.00 \\
\hline $\mathrm{D}_{\mathrm{pi} 2 J, 2}$ & $\mathrm{kHz}$ & $40.36(47)$ & & $18.78(34)$ & \\
\hline $\mathrm{D}_{\mathrm{pi} 2 K, 2}$ & $\mathrm{kHz}$ & $-206.5(61)$ & & $-343(24)$ & \\
\hline$\sigma^{[e]}$ & $\mathrm{kHz}$ & 2.4 & & 2.6 & \\
\hline$N^{[f]}$ & & 280 & & 105 & \\
\hline
\end{tabular}

[a] All parameters are given with one standard uncertainty in parentheses. Watson's $S$ reduction and $\mathrm{Ir}$ representation was used. [b] Vibrational ground state $B_{0}$ rotational constants and centrifugal distortion constants obtained by anharmonic frequency calculation at the MP2/6-311++G(d,p) level of theory. [c] Not fitted, set to zero. [d] Fixed due to symmetry. [e] Standard deviation of the fit. [f] Number of lines.

constants from the rigid rotor fit. The angles $\delta$ between the internal rotor axes and the principal $a$ axis as well as the $V_{3}$ potentials were calculated by $a b$ initio. The LAM splittings are relatively small (up to $20 \mathrm{MHz}$ ), which simplified the assignment. Some rotational transitions exhibited very small splittings down to a few $\mathrm{kHz}$. The assignment of those transitions was quite challenging because different torsional species could not be easily distinguished. Finally, 280 torsional transitions with $J \leq 10$ were assigned and fitted to a standard deviation of $2.4 \mathrm{kHz}$ using the program XIAM. The results of the fits are given in Table 1.

After the trans conformer was assigned, many lines with low intensities remained in the scan which presumably belong to the cis conformer with the calculated dipole moment components of $2.26 \mathrm{D}, 4.04 \mathrm{D}$, and $0.00 \mathrm{D}$ in $a-, b$-, and $c^{-}$ direction, respectively. Also in this case, the spectrum contains only $a$ - and $b$-type transitions and $b$-type lines should be more intense. Surprisingly, the $J=5 \leftarrow 4$ and $6 \leftarrow 5$ a-type transitions could be assigned first and they were more intense than most of the $b$-type lines. For the cis conformer, 105 torsional transitions up to $J=8$ were measured and fitted to a standard deviation of $2.6 \mathrm{kHz}$ (see Table 1). The frequency lists of both, the trans and the cis conformer, are available in Table S-4 in the Supporting Information.

\section{Results and Discussion}

The three linear combinations of the rotational constants $B_{J}=$ $1 / 2(B+C), B_{K}=A-1 / 2(B+C), B_{-}=1 / 2(B-C)$ and the centrifugal 
distortion constants $D_{J}, D_{J K}, D_{K}, d_{1}, d_{2}$ were determined with very high accuracy for both conformers. The $V_{3}$ potentials, the angles $\delta$ between the internal rotor axes and the principal a axis, the internal rotation constant, and two higher order parameters $D_{\text {piz }}$ and $D_{p i 2} K$ were also fitted.

The experimentally deduced rotational constants are compared with those from quantum chemical calculations. Best agreements can be found with the DFT method using the functionals M06-2X and B3LYP for the trans and the cis conformer, respectively, as shown in Table S-2 in the Supporting Information. All levels of theory yield quite reasonable $B$ and $C$ rotational constants, while the $A$ rotational constant has larger deviations. We note that all predicted rotational constants reported in Table S-2 refer to the equilibrium $r_{e}$ structure, while the experimentally deduced rotational constants to the vibrational ground state $r_{0}$ structure. Theoretical rotational constants of the ro structure can be obtained by anharmonic frequency calculation (given in Table 1). Surprisingly, they show larger deviations to the experimental values compared to those of the $r_{e}$ structure. This is probably due to error compensations. For more details, the vibration-rotation constants for each vibrational mode are given in Table S-5 in the Supporting Information.

Because of the strong correlation between $V_{3}$ and $F_{0}$ in the fit shown in Table 1, we carried out a fit with $F_{0}$ fixed to the $a b$ initio values (given in Table S-6). The $V_{3}$ terms, which depend on the assumed values of $F_{0}$, as well as the higher order parameters $D_{\text {pizJ }}$ and $D_{\text {pi2K }}$ change slightly. The $V_{6}$ term cannot be fitted independently, because only transitions in the torsional ground state are available, and we set it to zero. Since the $a b$ initio results predict no significant $V_{6}$ contributions (see section 2 ), we expect that the experimental values of the $V_{3}$ terms are sufficiently accurate.

The calculated centrifugal distortion constants obtained by anharmonic frequency calculations with the MP2 and B3LYP methods and the basis set $6-311++G(d, p)$ are in good agreement with the experimental values except for the parameter $D_{K}$ of the trans conformer (see Table 1). Since the planar five-membered ring frame of AMF is quite rigid, all centrifugal distortion constants are small. We recognized a relatively strong correlation between the parameters $B_{K}$ and $D_{K}$ in the fit of the trans conformer, if $F_{0}$ is fixed to its $a b$ initio values (see Table S-7 in the Supporting Information). This correlation decreases slightly if $F_{0}$ is fitted (as in the fit shown in Table 1 ; for the correlation matrices see also Table S-7), and the $D_{K}$ value is closer to the predicted value.

The barrier heights of the ring methyl top are $369.78(25) \mathrm{cm}^{-1}$ and $356.47(39) \mathrm{cm}^{-1}$ for the trans and the cis conformer, respectively, which are very similar. Obviously, the orientation of the acetyl group does not significantly affect the torsional barrier of the ring methyl group. On the other hand, the barrier heights are clearly lower than that found in 2-methylfuran $\left(416.2 \mathrm{~cm}^{-1}\right)$ [14], showing that the presence of the acetyl methyl group with its negative mesomeric effect and the possibility for an extended $\pi$-conjugation might decrease the barrier of the ring methyl group.

The barrier to internal rotation of the acetyl methyl top is quite different in two conformers. We found a value of $307.78(59) \mathrm{cm}^{-1}$ for the trans and $212.71(30) \mathrm{cm}^{-1}$ for the cis
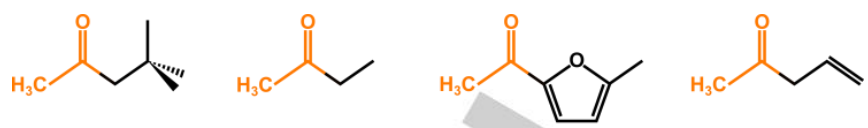

(1) $173.539(36)$

(2) $183.1702(89)$

(3) $212.71(30)$

(4) $225.57(10)$

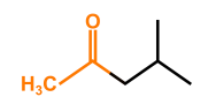

(5) $250.3(19)$ (6) $264.7(8)$ (7) $307.78(59)$

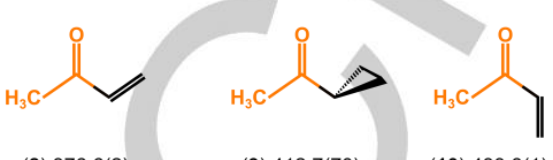

$\begin{array}{lll}\text { (8) } 376.6(2) & \text { (9) } 412.7(70) & \text { (10) } 433.8(1)\end{array}$

Figure 8. Torsional barriers of the acetyl methyl groups in ketones (in $\mathrm{cm}^{-1}$ ) (1) Methyl neopentyl ketone [13], (2) methyl ethyl ketone [14], (3) cis AMF (this work), (4) allyl acetone [15], (5) methyl isobutyl ketone [16], (6) acetone [17], (7) trans AMF (this work), (8) syn-periplanar-methyl vinyl ketone [18], (9) cyclopropyl methyl ketone [19], (10) anti-periplanar-methyl vinyl ketone [18].

conformer. From many previous investigations, it is known that the torsional barrier of the acetyl methyl group in ketones depends strongly on the substitution at the other side of the carbonyl group, as can be seen in Figure 8 [15-21]. This behavior in ketones, where the substituent on the other side of the carbonyl group causes the acetyl methyl barrier to vary over a wide range without any apparent trends, is in strong contrast to the acetates, which can be divided in classes with predictable barrier heights [22]. For example in $\alpha, \beta$-saturated alkyl acetates, the barrier to internal rotation is approximately $100 \mathrm{~cm}^{-1}$ and remains largely invariant [23,24]. This value cannot be easily predicted when electronic effect exists, e.g. by the presence of double bonds or lone electron pairs, which can contribute in a conjugated system. In unsaturated acetates like vinyl acetate [25] and isopropenyl acetate [26], $\pi$ electron conjugation, extending from the vinyl double bond to the ester group, is possible. The barriers are significantly different and also differ from those found for saturated acetates. In some acetamides such as $\mathrm{N}$-ethylacetamide [27] or N,N-diethylacetamide [5], it turns out that the barriers of the acetyl methyl group are strongly affected by the electronic effect from the lone electron pair and the substituents attached to the nitrogen. We note that though AMF is different from other ketones because of the aromatic ring, the barrier height in its both conformers is not unusually larger or smaller. Similar to the case of N,N-diethylacetamide [5] and methyl vinyl ketone [20], the barrier height of the acetyl methyl rotor depends strongly on the orientation of the substituent on the other side of the carbonyl group. Further conclusions cannot be drawn since too few ketone examples are available at the moment.

The calculated torsional barriers of the ring methyl group at different levels of theory are $297-501 \mathrm{~cm}^{-1}$ and $283-477 \mathrm{~cm}^{-1}$ for trans and the cis conformer, respectively, which are in the same order of magnitude of the experimental $V_{3}$ potentials. For the acetyl methyl top, we found $162-349 \mathrm{~cm}^{-1}$ for the trans and $71-311 \mathrm{~cm}^{-1}$ for the cis conformer. The best agreement 
between the calculated and experimental barriers were found for both conformers and both methyl groups at the M06-2X/6$311++\mathrm{G}(3 \mathrm{df}, 2 \mathrm{pd})$ level of theory (see Table S-2).

The inertial defects $\Delta_{c}=\left(I_{c}-l_{a}-I_{b}\right)=-6.469$ and -6.580 $\mathrm{u} \AA^{2}$ of the trans and cis conformers, respectively, confirm that the heavy atom skeleton is planar with two pairs of hydrogen atoms out of plane. These values are almost the same as that found in other planar molecules containing two methyl groups with comparable barrier heights, e.g. methyl acetate $\left(\Delta_{c}=\right.$ $\left.-6.315 \cup \AA^{2}\right)$ [28] and the syn and anti conformers of 3,4dimethylbenzaldehyde $\left(\Delta_{c}=-6.452\right.$ and $-6.469 \mathrm{u} \AA^{2}$, respectively) [29].

After the assignment of the two conformers, only very few weak lines remained in the broadband scan. We thus concluded that water complexes or dimers were not present under our measurement conditions.

\section{Conclusion}

Two conformers of 2-acetyl-5-methylfuran exhibiting the internal rotations of two non-equivalent methyl groups were successfully assigned under molecular beam conditions using a combination of Fourier-transform microwave spectroscopy and quantum chemistry. The above mentioned intramolecular dynamics causes splittings of all rotational transitions into five different torsional species. The torsional barriers of the ring methyl top are similar in both conformers; those of the acetyl methyl group are on the other hand quite different, which is probably because of the electronic rather than the steric effect. Quantum chemical calculations yielded reasonable structural parameters, but the predicted barrier heights are not yet sufficiently accurate.

\section{Experimental Section}

The rotational spectra were measured with a molecular beam Fourier transform microwave spectrometer operating in the frequency range from 2 to $26.5 \mathrm{GHz}$ [30]. AMF was purchased from TCl Deutschland GmbH, Eschborn, Germany with a stated purity of over $97 \%$ and used without further purification. The sample was placed on a $5 \mathrm{~cm}$ piece of a pipe cleaner inside a stainless steel tube mounted upstream the nozzle. Helium was flown over the sample at a pressure of approximately $200 \mathrm{kPa}$, the helium-substance mixture was expanded into the cavity.

\section{Acknowledgements}

V. V. thanks the Fonds der Chemischen Industrie $(\mathrm{FCl})$ for a Ph.D. fellowship. Simulations were performed with computing resources granted by JARA-HPC from the RWTH Aachen University under the project jara0124.

Keywords: internal rotation $•$ large amplitude motion $\cdot$ rotational spectroscopy $\cdot$ quantum chemical calculation

[1] L. J. Farmer, D. S. Mottram, F. B. Whitfield, J. Sci. Food Agric. 1989, 49 347-368.

[2] W. Fan, M. C. Quian, J. Agric. Food Chem. 2006, 54, 2695-2704.
[3] V. Varlet, C. Knockaert, C. Prost, T. Serot, J. Agric. Food Chem. 2006, 54, 3391-3401.

[4] D. Ryan, R. Shellie, P. Tranchida, A. Casilli, L. Mondello, P. Marriott, J. Chromatogr. A 2004, 1054, 57-65.

[5] R. Kannengießer, S. Klahm, H. V. L. Nguyen, A. Lüchow, W. Stahl, J. Chem. Phys. 2014, 141, 204308.

[6] V. Van, W. Stahl, H. V. L. Nguyen, Phys. Chem. Chem. Phys. 2015, 17, 32111-32114.

[7] M. J. Frisch, G. W. Trucks, H. B. Schlegel, G. E. Scuseria, M. A. Robb, J. R. Cheeseman, G. Scalmani, V. Barone, B. Mennucci, G. A Petersson, H. Nakatsuji, M. Caricato, X. Li, H. P. Hratchian, A. F. Izmaylov, J. Bloino, G. Zheng, J. L. Sonnenberg, M. Hada, M. Ehara, K. Toyota, R. Fukuda, J. Hasegawa, M. Ishida, T. Nakajima, Y. Honda, O. Kitao, H. Nakai, T. Vreven, J. A., Jr. Montgomery, J. E. Peralta, F. Ogliaro, M. Bearpark, J. J. Heyd, E. Brothers, K. N. Kudin, V. N. Staroverov, R. Kobayashi, J. Normand, K. Raghavachari, A. Rendell, J. C. Burant, S. S. Iyengar, J. Tomasi, M. Cossi, N. Rega, J. M. Millam, M. Klene, J. E. Knox, J. B. Cross, V. Bakken, C. Adamo, J. Jaramillo, R. Gomperts, R. E. Stratmann, O. Yazyev, A. J. Austin, R. Cammi, C. Pomelli, J. W. Ochterski, R. L. Martin, K. Morokuma, V. G. Zakrzewski, G. A. Voth, P. Salvador, J. J. Dannenberg, S. Dapprich, A. D. Daniels, O. Farkas, J. B. Foresman, J. V. Ortiz, J. Cioslowski, D. J. Fox, Gaussian 09, Revision A.02, Gaussian, Inc., Wallingford CT, 2009.

[8] R. Ditchfield, W. J. Hehre, J. A. Pople, J. Chem. Phys. 1971, 54, 724728.

[9] T. H. Dunning Jr., J. Chem. Phys. 1989, 90, 1007-1023.

[10] H. Dreizler, Z. Naturforsch. 1961, 16a, 1354-1367.

[11] H. B. Schlegel, J. Comput. Chem. 1982, 3, 214-218.

[12] W. Gordy, R. L. Cook, Microwave Molecular Spectra, John Wiley \& Sons, New York 1984, $3^{\text {rd }}$ edition.

[13] H. Hartwig, H. Dreizler, Z. Naturforsch. A 1996, 51, 923-932.

[14] W. G. Norris, L. C. Krisher, J. Chem. Phys. 1969, 51, 403-406.

[15] Y. Zhao, J. Jin, W. Stahl, I. Kleiner, J. Mol. Spectrosc. 2012, 281, 4-8.

[16] H. V. L. Nguyen, V. Van, W. Stahl, I. Kleiner, J. Chem. Phys. 2014, 140, 214303.

[17] L. Tulimat, H. Mouhib, W. Stahl, I. Kleiner, J. Mol. Spectrosc. 2015, 312, 46-50.

[18] Y. Zhao, W. Stahl, H. V. L. Nguyen, Chem. Phys. Lett. 2012, 545, 9-13.

[19] R. Peter, H. Dreizler, Z. Naturforsch. 1965, 20a, 301-312.

[20] D. S. Wilcox, A.J. Shirar, O. L. Williams, B. C. Dian, Chem. Phys. Lett. 2011, 508, 10-16.

[21] P. L. Lee, R. H. Schwendeman, J. Mol. Spectrosc. 1972, 41, 84-94.

[22] A. Jabri, V. Van, H. V. L. Nguyen, W. Stahl, I. Kleiner, ChemPhysChem. 2016, DOI: 10.1002/cphc.201600265.

[23] D. Jelisavac, D. C. Cortés-Gómez, H. V. L. Nguyen, L. W. Sutikdja, W. Stahl, I. Kleiner, J. Mol. Spectrosc. 2009, 257, 111-115.

[24] L. W. Sutikdja, W. Stahl, V. Sironneau, H. V. L. Nguyen, I. Kleiner, manuscript accepted in Chem. Phys. Lett. 2016.

[25] H. V. L. Nguyen, A. Jabri, V. Van, W. Stahl, J. Phys. Chem. A 2014, 118, 12130-12136.

[26] H. V. L. Nguyen, W. Stahl, J. Mol. Spectrosc. 2010, 264, 120-124.

[27] R. Kannengießer, M. J. Lach, W. Stahl, H. V. L. Nguyen, ChemPhysChem, 2015, 9, 1906-1911.

[28] M. Tudorie, I. Kleiner, J. T. Hougen, S. Melandri, L. W. Sutikdja, W Stahl, J. Mol. Spectrosc. 2011, 269, 211-225.

[29] M. Tudorie, I. Kleiner, M. Jahn, J.-U. Grabow, M. Goubet, O. Pirali, J. Phys. Chem. A 2013, 117,13636-13647.

[30] J.-U. Grabow, W. Stahl, H. Dreizler, Rev. Sci. Instrum. 1996, 67, 4072 4084 
Entry for the Table of Contents (Please choose one layout)

Layout 1:

\section{ARTICLE}

Hard to guess - The torsional barriers of methyl groups

Two different conformers of 2-acetyl5-methylfuran with two non-equivalent methyl groups each were analyzed by microwave spectroscopy. The barriers to internal rotation were accurately determined. Quantum chemistry yields only the correct order of magnitude while chemical intuition fails completely.

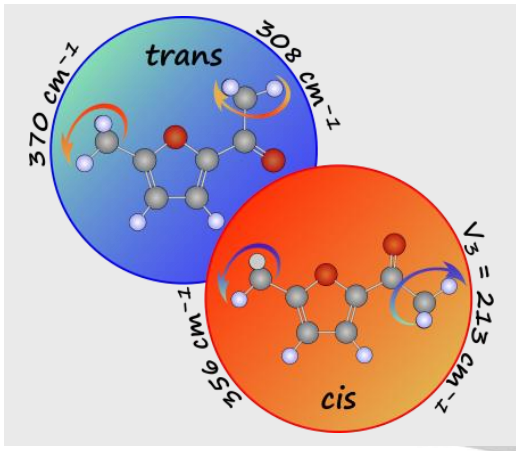

Vinh Van, Wolfgang Stahl, Ha Vinh Lam Nguyen*

Page No. - Page No.

The structure and torsional dynamics of two methyl groups in 2-acetyl-5methylfuran as observed by

microwave spectroscopy 